\title{
Demonstration of Josephson effect submillimeter wave sources with increased power
}

\author{
Siyuan Han, Baokang Bi, Wenxing Zhang, and J. E. Lukens \\ Department of Physics, SUNY at Stony Brook, Stony Brook, New York 11794
}

(Received 18 October 1993; accepted for publication 13 December 1993)

\begin{abstract}
A submillimeter wave source based on a new design using Josephson junction arrays has been developed and tested. The maximum rf power, delivered to a $68 \Omega$ load and detected on chip, was $47 \mu \mathrm{W}$ at $394 \mathrm{GHz}$. Significant power was detected at a number of frequencies from 300 to 500 $\mathrm{GHz}$ where the power was $10 \mu \mathrm{W}$. The observed power at the designed operating frequency near $400 \mathrm{GHz}$ is consistent with all 500 junctions in the series biased array delivering current in phase to the loads. This is in agreement with simulations of smaller arrays of the same design. The linewidth, inferred from the measured resistance at the point of maximum power, with $T=4.2 \mathrm{~K}$, is less than $1 \mathrm{MHz}$. The minimum inferred linewidth near $400 \mathrm{GHz}$, at somewhat lower power, is about $100 \mathrm{kHz}$.
\end{abstract}

Many submillimeter wave (SMW) frequency applications, such as radio astronomy and high speed communication, require a compact SMW source capable of delivering $\mathrm{rf}$ power in the range of $10-100 \mu \mathrm{W}$. For frequencies above $300 \mathrm{GHz}$, the lack of compact sources is particularly serious requiring increasingly inefficient multiplication of millimeter wave sources. Lately there has been a substantial effort to develop Josephson effect sources, which can work to at least $1 \mathrm{THz}, \stackrel{1}{-}$ for applications in the SMW..$^{2-4}$ The most promising results, recently obtained, have come from three types of sources: long single junctions flux flow oscillators, ${ }^{2}$ various types of two-dimensional arrays of small junctions, ${ }^{3}$ and onedimensional (1D) small junction arrays, ${ }^{4}$ discussed here. In general a power of about $1 \mu \mathrm{W}$ is obtained from these sources, although in many cases the source impedance is less than $1 \Omega$. The highest of power previously reported from a Josephson effect oscillator has been $7 \mu \mathrm{W}$ at $\sim 300 \mathrm{GHz}$ by Wan $e t$ al. $^{4}$ using a 1D array. Here we report results from an improved design for Josephson SMW sources-distributed linear arrays of 500 Josephson junctions with series dc biasing-generating an rf power of $47 \mu \mathrm{W}$ around $394 \mathrm{GHz}$ and $10 \mu \mathrm{W}$ at $500 \mathrm{GHz}$ into an on-chip load of $68 \Omega$.

Small resistively shunted junctions (RSJ), described by the RSJ model, ${ }^{5}$ are characterized by a critical current $I_{c}$ and a shunt resistance $R_{J}$, and generate a peak if voltage $V_{\mathrm{rf}}=\gamma V_{c}\left(V_{c} \equiv I_{c} R_{J}\right)$ with a source impedance $Z_{s}$ at the Josephson frequency, $f_{J}=483.6 \mathrm{GHz} / \mathrm{mV}$. For bias voltage $V>V_{c}, \gamma \rightarrow 1$, and $Z_{s} \rightarrow R_{J}$. The junctions are placed in a superconducting microstrip and interact via the rf current, $I_{\text {rf }}$ generated in the microstrip by the Josephson oscillations. For properly designed arrays ${ }^{6}$ the rf voltages across the junctions can be made to add in series. This will be true independent of the relative polarities, along the microstrip, of the junctions' biases, i.e., the dc voltage can sum to $N V_{c}$ (series bias) or to zero (parallel bias). The power from such an array (see Fig. 1 ) is then just

$$
P_{N}=\kappa \frac{\left(\gamma N V_{c}\right)^{2} R_{L}}{2\left(N R_{J}+R_{L}\right)^{2}}
$$

Here $N$ is the number of $\mathrm{rf}$ series elements in the array, and
$R_{L}$ is the load impedance seen by the array. $\kappa=1$ is the ideal case, but is in general somewhat less than unity due to losses in the coupling structure or imperfect phase alignment of the junctions. For $1 \mathrm{D}$ arrays [Figs. 1(a)-1(c)], $N$ is just the number of junctions in the array. For 2D arrays [Fig. 1(d)] of the types so far reported, the if current flow is ideally along rows of junctions, with the phase of the rf current transverse to this flow (i.e., across a column) being constant. Equation (1) can then be applied to 2D arrays as well if one replaces $N$ by $N_{s}$, the number of series elements (i.e., columns) in the array and $R_{J}$ by $R_{J s}$ the resistance of a series element. The power from an array matched to the load, i.e., with $N_{s} R_{J s}=R_{L}$, is $P_{N} \simeq\left(N_{s} V_{c}\right)^{2} / 8 R_{L}$, and can in principle be increased just by increasing $N_{s}$ while keeping $N R_{J}$ and $V_{c}$ constant. This implies that $I_{c s} \propto N_{s}$ and $P_{N} \simeq R_{I} I_{c s}^{2} / 8$.

Increasing the source power, therefore, requires increasing $I_{c s}$, adding together enough series elements to match the array impedance to the load and maintaining phase coherence throughout the array. In practice, there are two essential limitations to this increase. First, when $I_{c s}$ becomes greater than several milliamps, there exists the potential for flux flow instabilities which would dissipate power internally in both single junctions and in the columns of $2 \mathrm{D}$ arrays. It is not yet clear whether there is an advantage to $1 D$ or $2 D$ arrays for maximizing $I_{c s}$ while maintaining phase stability. The second problem, common to both $1 \mathrm{D}$ and $2 \mathrm{D}$ arrays, is that when the length of the array becomes an appreciable fraction of the wavelength, care must be taken to maintain the proper phase relationship between the junction oscillations and the rf current throughout the array. This is required so that all junctions phase lock in a stable manner and provide current to the load with the same phase. Especially for the SMW source, this problem must be solved to permit the use of enough junctions to obtain power levels much above a microwatt. It is this second problem which we address in this paper.

Fully coherent 1D arrays have been demonstrated using both a lumped circuit design ${ }^{6}$ [Fig. 1(a)], where the array length $L<\lambda / 10$ ( $\lambda$ is the wavelength in the microstrip coupling the junctions), or the so-called quasilumped design where the junctions' spacing is $\lambda .^{4}$ The number of junctions 


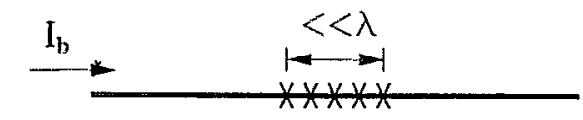

(a)

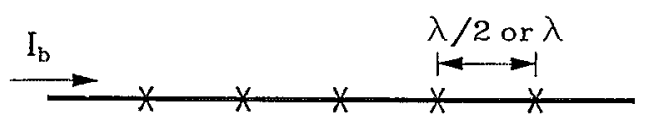

(b)

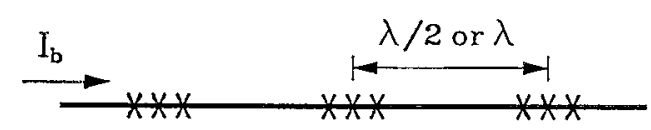

(c)

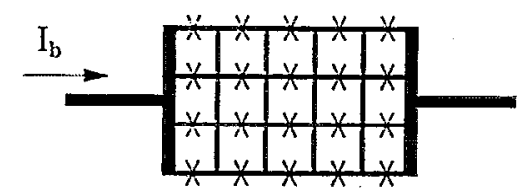

(d)

FIG. 1. Some typical structures for Josephson junction arrays: (a) 1D lumped array, (b) quasilumped array, (c) distributed array, and (d) 2D array. $\lambda$ is the wavelength at the primary operating frequency; $I_{b}$ is the dc bias current.

in both designs is severely limited in the SMW. A maximum of about $N=10$ can be used in the SMW for lumped arrays made with standard fabrication technologies. For quasilumped arrays, losses in the microstrip coupling the junctions as well as the larger array size are limitations. Much denser packing can be achieved if the junctions can be distributed along the microstrip for a significant fraction of $\lambda$. Figure 1(c) shows such a structure where groups of $M$ junctions are placed at intervals of $\lambda$ or $\lambda / 2$ along the microstrip. Our computer simulation shows that for same $N$ and $M$ arrays with $\lambda / 2$ interlump distance has a wider tuning range.

Distributed arrays (shown in Fig. 2) of 500 junctions have been designed, fabricated, and tested for SMW generation. Resistively shunted $\mathrm{Nb} / \mathrm{AlO}_{x} / \mathrm{Nb}$ tunnel junctions are placed in groups of ten junctions $(M=10)$. with adjacent junctions in the group separated by $10 \mu \mathrm{m}$. Adjacent groups are separated by one wavelength at the designed primary operating frequency of $\sim 390 \mathrm{GHz}$. Load resistors and Josephson junction detectors to measure the if currents are placed at both ends of the array. The arrays were fabricated at IBM using planarized all-refractory technology for superconductivity ${ }^{7}$ with high critical current density $\left(J_{c} \approx 40-60 \mathrm{kA} / \mathrm{cm}^{2}\right) \mathrm{Nb} / \mathrm{AlO}_{x} / \mathrm{Nb}$ trilayers made at AT\&T Bell Laboratory. $700 \mathrm{~nm}$ of thermally evaporated $\mathrm{SiO}$ was used as the dielectric between the $\mathrm{Nb}$ microstrip and the 300 $\mathrm{nm} \mathrm{Nb}$ ground plane, which was placed on top of the array. Each load resistor and detector junction has independent dc bias leads allowing their parameters to be determined using four terminal measurements. $R_{J}$ is measured by suppressing the junctions' critical current with a magnetic field. The ca-

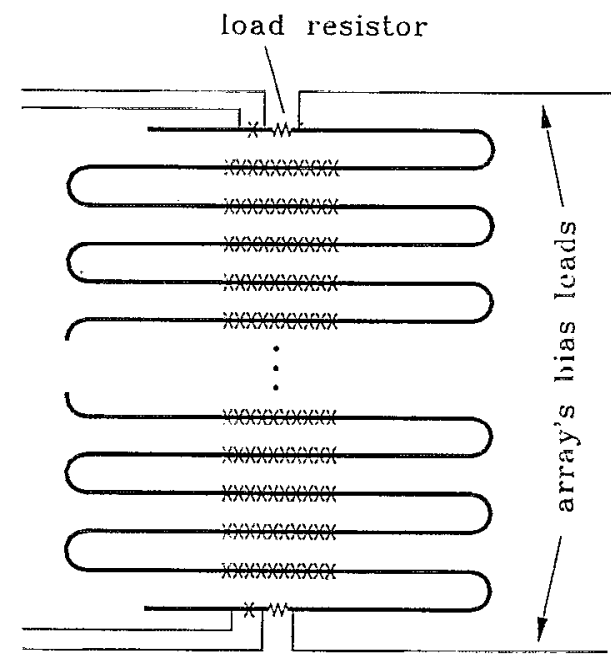

detector's

bias leads

FIG. 2. Schematics of the $N=500, M=10$ serial biased 1D distributed Josephson junction array for SMW generation.

pacitance of the $6 \mu \mathrm{m}^{2}$ junctions, including parasitic capacitance, is estimated from the geometry and the specific capacitance of $46 \mathrm{fF} / \mu \mathrm{m}^{2}$ to be $\sim 290 \mathrm{fF}$. The parasitic inductance $L$ associated with the external shunt resistor is $0.18 p \mathrm{H}$ estimated from the physical dimensions of the shunt. The junctions in the array are biased in series using a common dc current. This requires a higher degree of junction uniformity, but is important for larger arrays. For example, the arrays studied here would have required a bias current of over $2 \mathrm{~A}$ for parallel bias instead of the $5 \mathrm{~mA}$ actually used.

The coherent rf current generated by the array is measured by the detector junctions placed after the lumped resistor loads. The magnitude of the $n=1$ Shapiro step ${ }^{8}$ is used to obtain the amplitude of the coherent rf current $I_{\text {rf }}$ through the detector junction and the load resistor by fitting the measured $I V$ curve to that of numerical simulations using measured junction parameters. For numcrical simulations, the detector junction has to be described by the resistively inductively and capacitively shunted junction model ${ }^{9}$ due to the rather large value of $L I_{c}$.

Five samples have been completed and tested for SMW generation. All delivered more than $20 \mu \mathrm{W}$ of rf power near $394 \mathrm{GHz}$ to loads of about $65 \Omega$. For the sample W11D8, the average critical current of the junctions in the array is $I_{c}=3.2$ $\mathrm{mA}$ and the average junction resistance is $R_{J}=0.38 \Omega$. The detector junctions have an $I_{c}=3.5 \mathrm{~mA}$, and a $R_{J}=0.38 \Omega$. The maximum power from the array occurs at $394 \mathrm{GHz}$; however, significant power is available at a number of frequencies in the $300-500 \mathrm{GHz}$ range with $10 \mu \mathrm{W}$ of power delivered to the loads at $500 \mathrm{GHz}$. Figure 3 shows the measured $I V$ curve of a detector around the $n=1$ Shapiro step with the array operating at $394 \mathrm{GHz}$ and $T=1.9 \mathrm{~K}$. This is compared with that of the detector simulation using the measured junction parameters and a rf current amplitude from the array of $I_{\mathrm{rf}}=1.18 \mathrm{~mA}$. The measured total load resistance is $68 \Omega$ for this sample giving a rf power, $I_{\mathrm{rf}}^{2} R_{L} / 2 \simeq 47 \mu \mathrm{W}$ at 


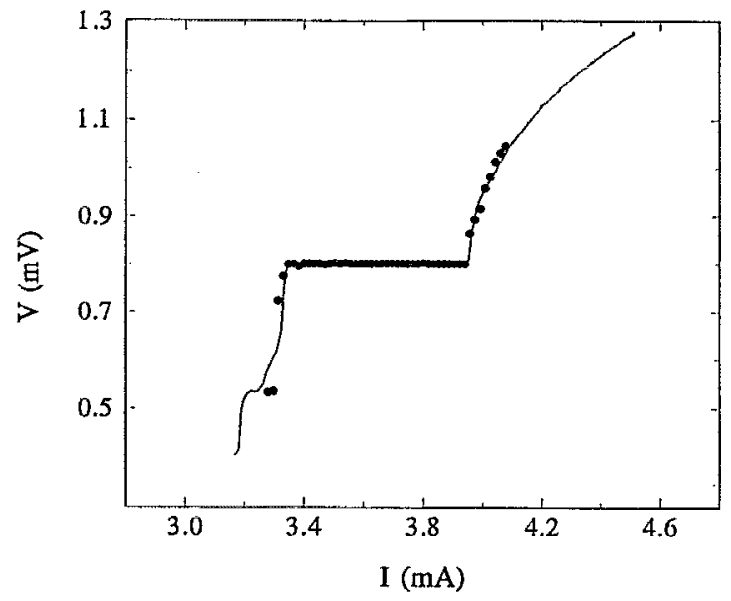

FIG. 3. The measured $I V$ curve (solid line) around the $n=1$ Shapiro step at $798 \mu \mathrm{V}$ of the detector junction on the sample W11D8 under $394 \mathrm{GHz}$ irradiation from the array and the $I V$ curve obtained from computer simulation (solid circles) using the RICSJ model. The parameters used for the stimulation are $I_{c}=3.47 \mathrm{~mA}, C=294 \mathrm{fF}, L=0.178 p \mathrm{H}, R_{f}=0.377 \Omega$, and $I_{\mathrm{rf}}=1.18 \mathrm{~mA}$. This rf current amplitude corresponds to a power of $47 \mu \mathrm{W}$ in the load of $68 \Omega$.

$394 \mathrm{GHz}$. Since the total array resistance is $190 \Omega$ (the actual junctions shunt resistors were somewhat greater than the design values) the maximum available power from this array (into a $190 \Omega$ load) would be $64 \mu \mathrm{W}$ at $394 \mathrm{GIJz}$.

The measured power of $\sim 50 \mu \mathrm{W}$ at $394 \mathrm{GHz}$ compares reasonably with the value of $96 \mu \mathrm{W}$ from Eq. (1), assuming perfect alignment of the junctions' phases and no transmission line losses (i.e., $\kappa=1$ ). However, $\kappa$ is in general always less than unity due to imperfect phase alignment and the losses in the coupling structure (in our case, a microstrip transmission line). The measured value of $\kappa=47 / 96 \approx 0.49$ at $394 \mathrm{GHz}$ can be entirely accounted for by a surface resistance of $7 \mathrm{~m} \Omega$, assuming nearly perfect phase alignment. Our computer simulations of an array with $N=100$ but otherwise identical in structure to that measured indeed show a negligible phase spread among the junctions. Although the surface resistance of the actual sample has not been measured, the value inferred from the rf power measurement ( 7 $\mathrm{m} \Omega$ ) is consistent with the measured surface resistance at about $400 \mathrm{GHz}$ in $\mathrm{Nb} / \mathrm{SiO} / \mathrm{Nb}$ microstrip resonators ${ }^{10}$ and compares favorably to that obtained by Cucolo et al. ${ }^{11}$

The second major advantage of the phase-locked array source is that the radiation linewidth can be substantially reduced since the phase locking suppresses the frequency modulation of the array due to the random noise sources associated with the junction shunt resistors. The linewidth of a series array of $N$ identical Josephson junctions is approximately given by ${ }^{6,12,13}$

$$
\begin{gathered}
\Delta f \simeq\left(\frac{2 \pi}{\Phi_{0}}\right)^{2}\left(\frac{k_{B} T}{\pi R_{J}}\right) \frac{R_{d}^{2}}{N}\left[1+2 \alpha^{2} x \operatorname{coth}(x)\right], \\
x \equiv \frac{h f}{2 k_{B} T},
\end{gathered}
$$

where $k_{B}$ is Boltzmann's constant, $h$ is Planck's constant, $\Phi_{0}$ is the magnetic flux quantum, $R_{d}$ is the dynamic resistance of an independent junction, and $\alpha \simeq 0.42$ is the down conversion coefficient. Equation (2) requires some modification in distributed arrays, since the strength of the locking can depend on the position of the junction. However, direct measurements of the linewidth ${ }^{13}$ in a small array consisting of two groups of junctions separated by $\lambda / 2$ show that, within a factor of $2-3$, Eq. (2) provides a reliable estimate of $\Delta f$. Using values of $R_{d}$ and $R_{J}$ as the average over the array, the implied linewidth at $394 \mathrm{GHz}$ is about $730 \mathrm{kHz}$ at $T=4.2 \mathrm{~K}$ decreasing to $475 \mathrm{kHz}$ at $1.6 \mathrm{~K}$, where $x \simeq 5.9$, so zero point fluctuations dominate. The value of $R_{d}$ varies with bias current giving a minimum implied linewidth of about $100 \mathrm{kHz}$ at $T=4.2 \mathrm{~K}$ near $375 \mathrm{GHz}$ but with a somewhat lower power level.

In summary, 500 junction Josephson effect arrays using dc series bias and a distributed junction layout have been designed, fabricated, and tested. The measured maximum coherent rf power coupled to a $68 \Omega$ load at $394 \mathrm{GHz}$ is $47 \mu \mathrm{W}$, implying an available power of $64 \mu \mathrm{W}$. A rf power of $10 \mu \mathrm{W}$ has also been measured at $500 \mathrm{GHz}$ from the array oscillator. At $4.2 \mathrm{~K}$ the linewidth near $400 \mathrm{GHz}$, calculated from Eq. (2) using the measured array parameters, is less than 730 $\mathrm{kHz}$.

This work is supported in part by BMDO-IST through the RADC and by ONR, with support for the sample fabrication provided by CSE, which is supported in part by ARPA. We gratefully thank R. E. Miller and W. H. Mallison at AT\&T for providing the $\mathrm{Nb} / \mathrm{AlO} / \mathrm{Nb}$ trilayer films and $\mathrm{M}$. Ketchen's group at IBM for fabricating the sample. We also thank A. W. Worsham and A. Jain for their valuable assistance and discussion.

${ }^{1}$ R. P. Robertazzi and R. A. Buhrman, IEEE Trans. Magn. MAG-25, 1384 (1989).

${ }^{2}$ Y. M. Zhang, D. Winkler, and T. Claeson, Appl. Phys. Lett. 62, 3195 (1993). V. P. Koshelets, A. V. Shchukin, and S. V. Shitov, IEEE Trans. Appl. Supercond. 3, 2524 (1993).

${ }^{3}$ S. P. Benz and C. J. Burroughs, Appl. Phys. Lett. 58, 2162 (1991); J. S. Martens, A. Pance, K. Char, L. Lee, S. Whiteley, and V. M. Hietala, ibid. 63, 1681 (1993).

${ }^{4}$ K. Wan, A. K. Jain, and J. E. Lukens, Appl. Phys. Lett. 54, 1805 (1989).

${ }^{5}$ D. E. McCumber, J. Appl. Phys. 39, 2113 (1968); W. C. Stewart, Appl. Phys. Lett. 12, 277 (1968).

${ }^{\kappa}$ A. K. Jain, K. K. Likharev, J. E. Lukens, and J. E. Sauvageau, Phys. Rep. 109, 309 (1984); J. E. Lukens, in Superconducting Devices, edited by S. T. Ruggiero and D. A. Rudman (Academic, San Diego, 1991), p. 135.

${ }^{7}$ M. B. Ketchen, D. Pearson, A. Kleinsasser, C.-K. Hu, M. Smyth, J. Logan, K. Stawiasz, M. Jaso, K. Petrillo, M. Manny, S. Basavaiah, S. Brodsky, S. B. Kaplan, W. J. Gallagher, and M. Bhushan, Appl. Phys. Lett. 59, 2609 (1991); R. E. Miller, W. H. Mallison, A. W. Kleinsasser, K. A. Delin, and E. M. Macedo, Appl. Phys. Lett. 63, 1423 (1993).

${ }^{8}$ S. Shapiro, Phys. Rev. Lett. 11, 80 (1963).

${ }^{9}$ S. Han, A. H. Worsham, and J. E. Lukens, IEEE Trans. Appl. Supercond. 3, 2489 (1993).

${ }^{10}$ B. Bi, K. Wan, W. Zhang, S. Han, and J. E. Lukens, IEEE Trans. Appl. Supercond. 1, 145 (1991).

${ }^{11}$ A. N. Cucolo, S. Pace, R. Vaglio, V. Lacquaniti, and G. Marullo, IEEE Trans. Magn. MAG-17, 812 (1981).

${ }^{12} \mathrm{~K}$. K. Likharev and V. K. Semenov, JFTP Lett. 15, 442 (1972)

${ }^{13}$ B. Bi, S. Han, and J. E. Lukens, Appl. Phys. Lett. 62, 2745 (1993). 